\title{
Mapping of a database to a new ontology of Moroccan bac post learner profile for the E-orientation system MMSyOrientation
}

Guerss Fatima Zahra ${ }^{*}$,

Douzi Khadijab,

Aitdaoud Mohammed ${ }^{\mathrm{c}}$, Kamal ElGuemmat ${ }^{d}$, Talbi Mohammede, Namir Abdelouahez ${ }^{f}$,

Suggested Citation:

Abstract

SE SE SE 
Humanities and Social Sciences.

\section{State of art}

Direct Mapping:

\section{SE}

b. Augmented Direct Mapping

c. Domain Semantics-Driven Mapping

a. Materialization data:

b. The mapping on demand: 
Humanities and Social Sciences.

a. Based access requests:

SE

S

S

b. Linked data:

E $\quad$ SE $\quad$ SE

$S$

S E

$\bullet$

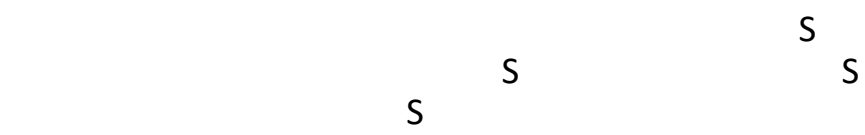

E

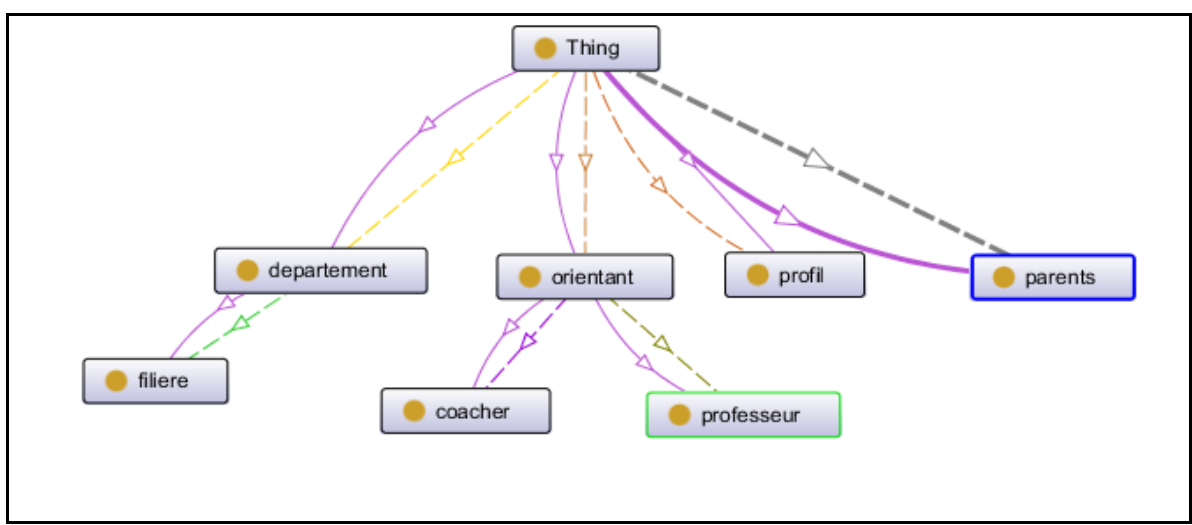

*http://www.w3.org/TR/rdf-sparql-query/ 
Humanities and Social Sciences.

Figure 1. Our former Ontology implemented within protected 2000 (Guerss F., Ait daoud M. \& al, 2015)

\section{Data Models}

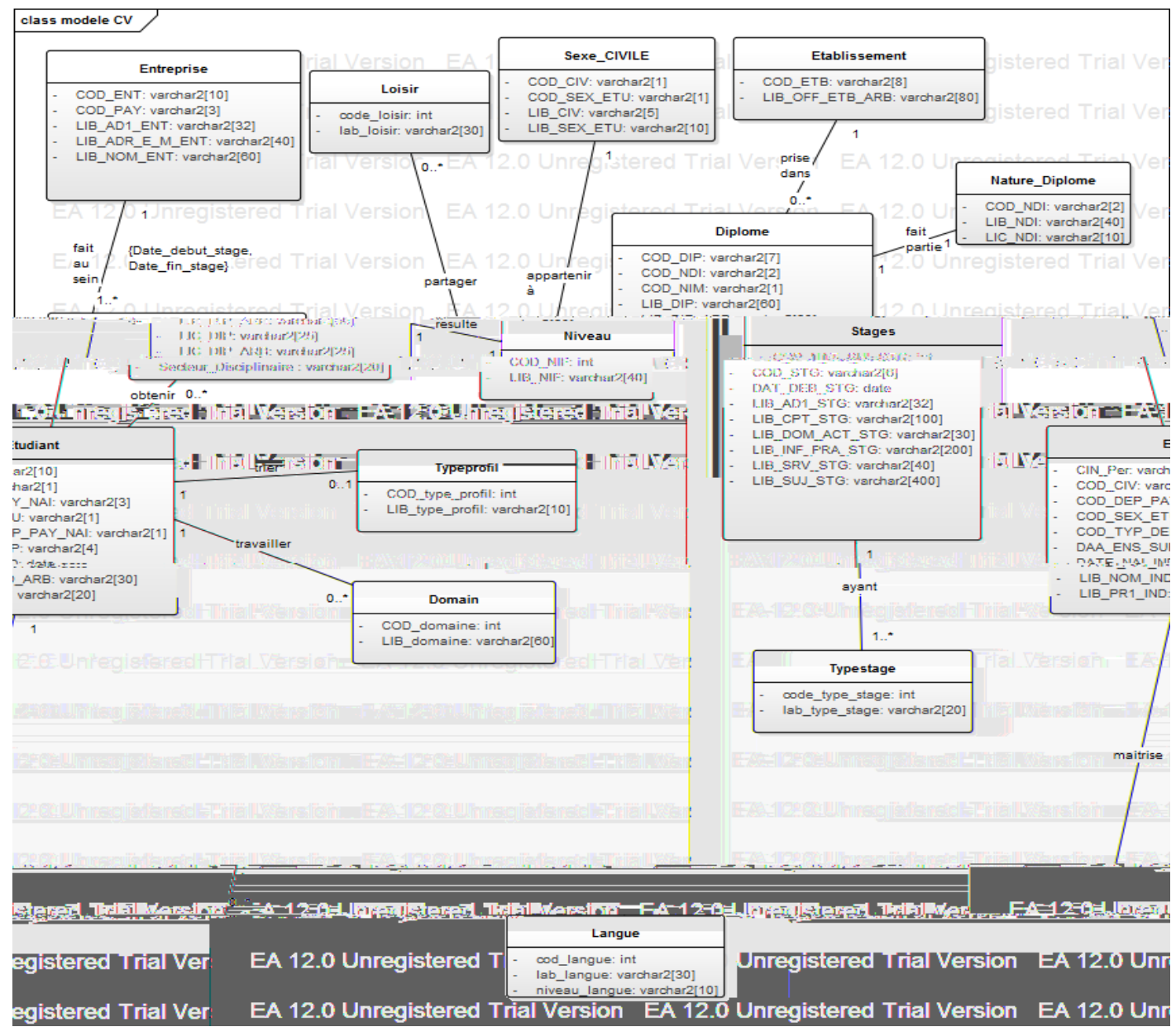

Figure 2. Class diagram of a model curriculum vitae 


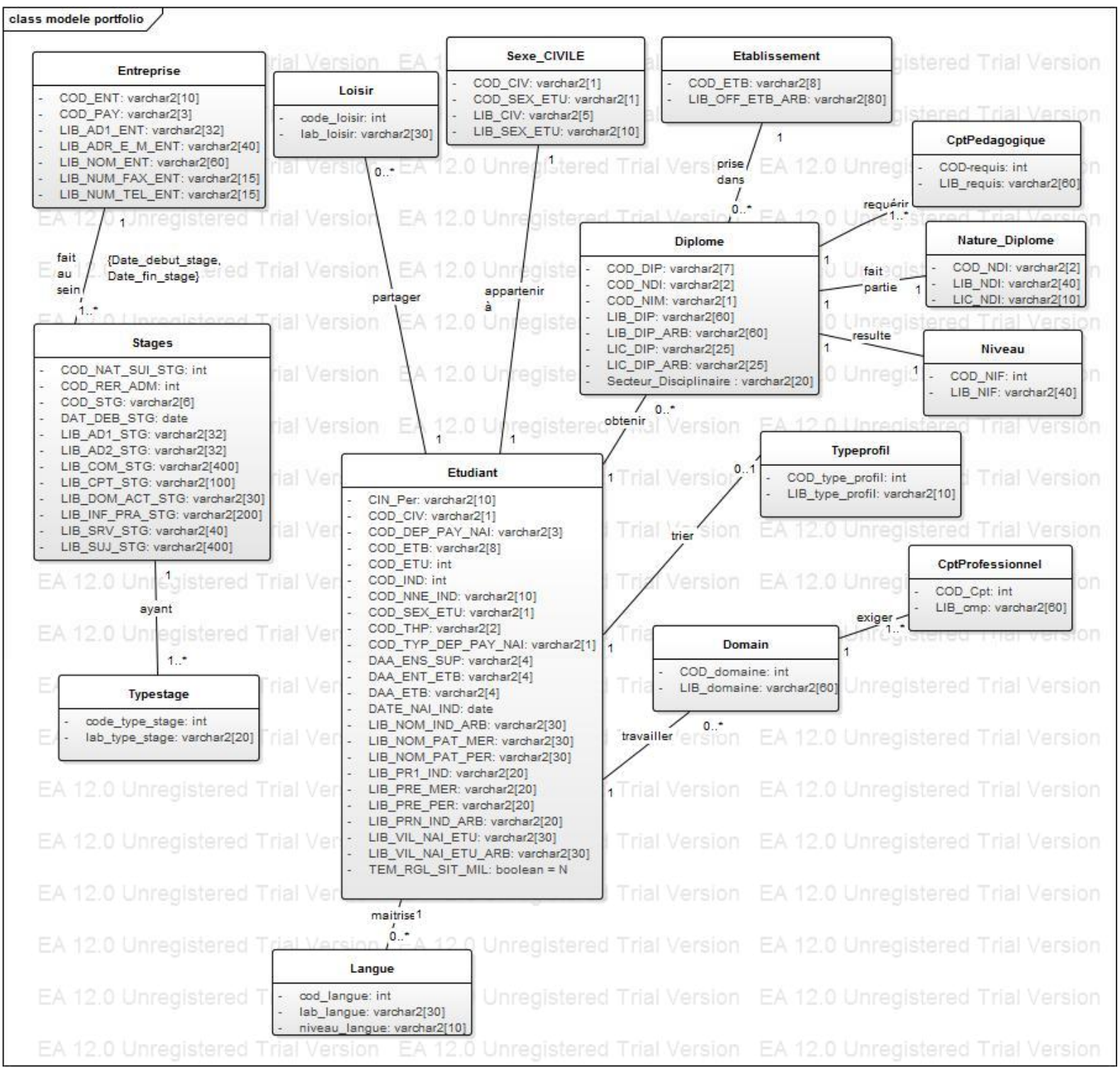

Figure 3. Class diagram of a model Portfolio 


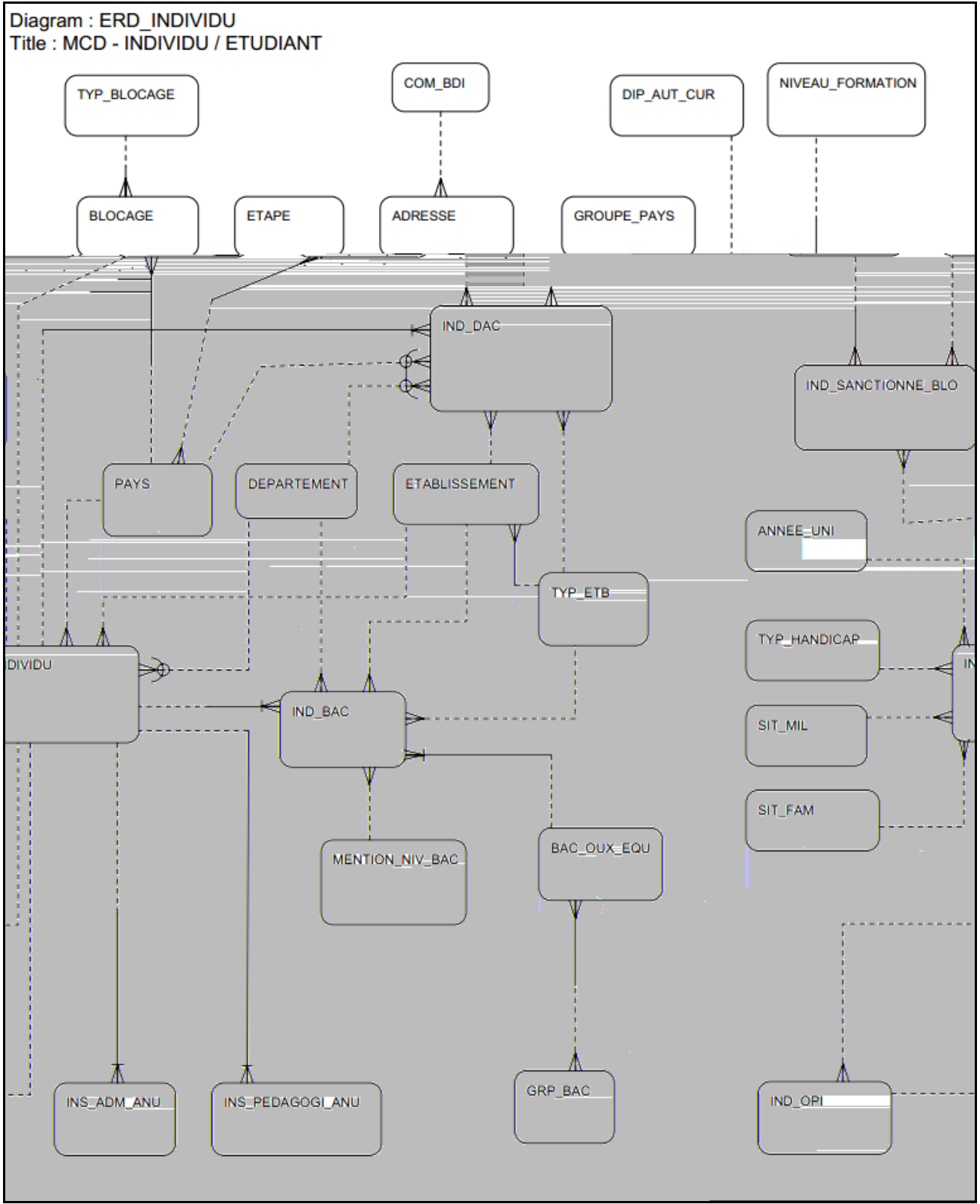

Figure 4. Conceptual data model of a student 
Humanities and Social Sciences.

\section{Approach}

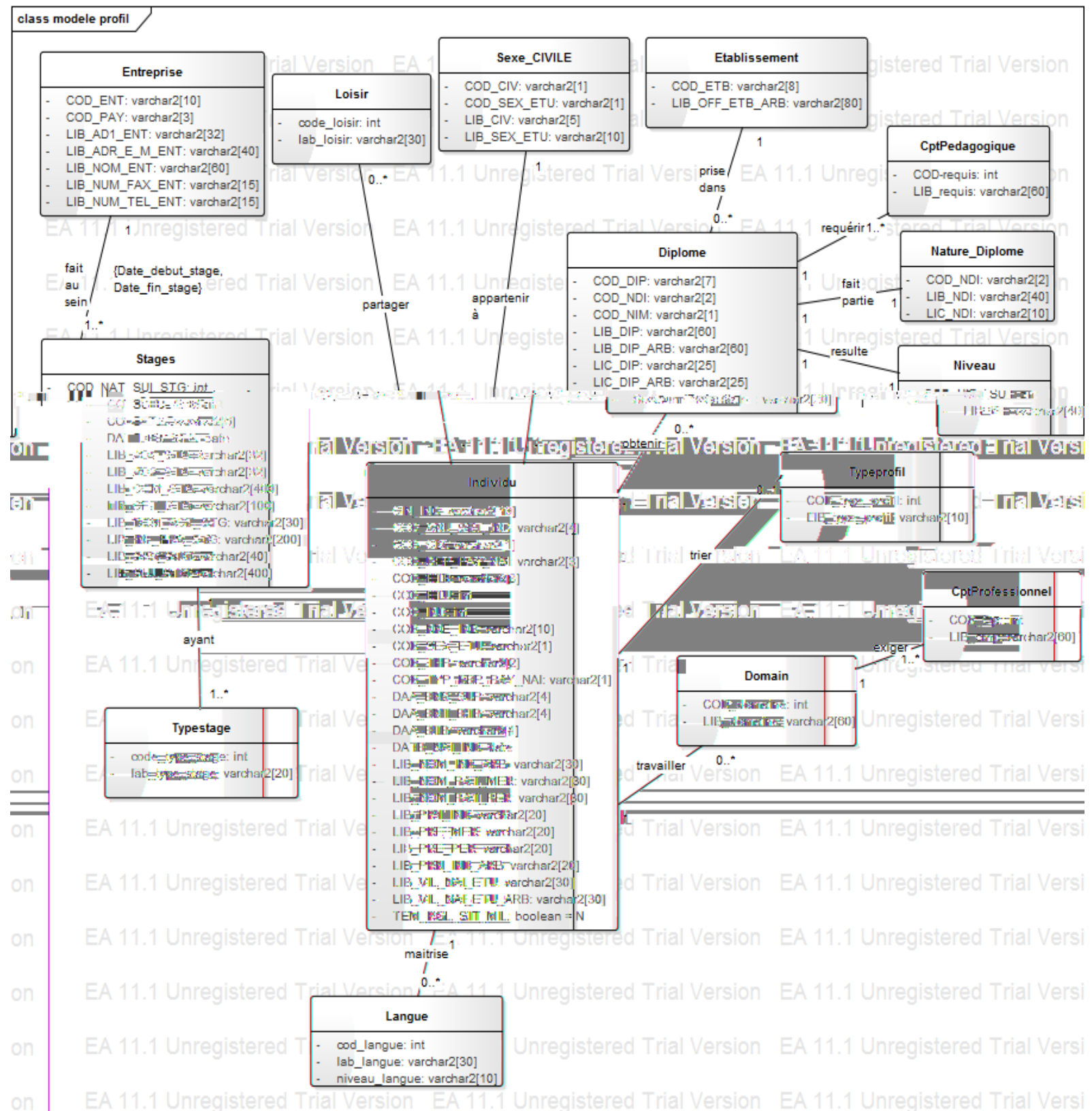

Figure 5. Conceptual data model of a student 
Humanities and Social Sciences.

SE SE 
Humanities and Social Sciences.

$\bullet$

S

SE $\quad S$

E

$\mathrm{E}$

E

E

E

E

E

E

E

E

E

E

E

E

E

E
S

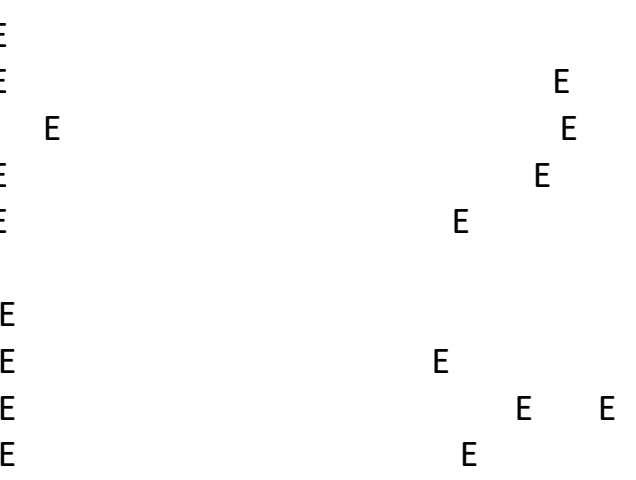

E

\section{Implementation}

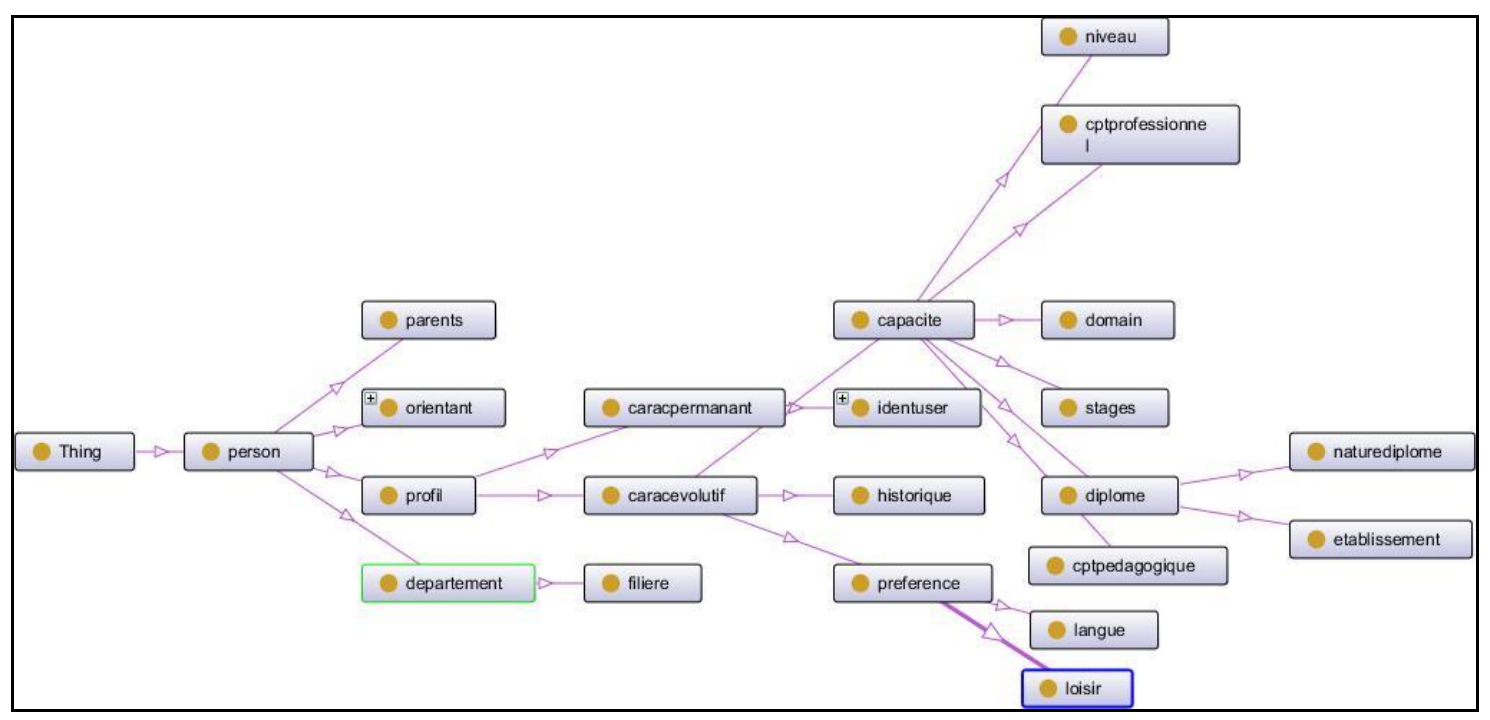

Figure 6. Orientation ontology domain (Onto E-orientation) (Guerss F., Ait daoud M. \& al, 2015)

\section{Conclusion}


Humanities and Social Sciences.

\section{References}

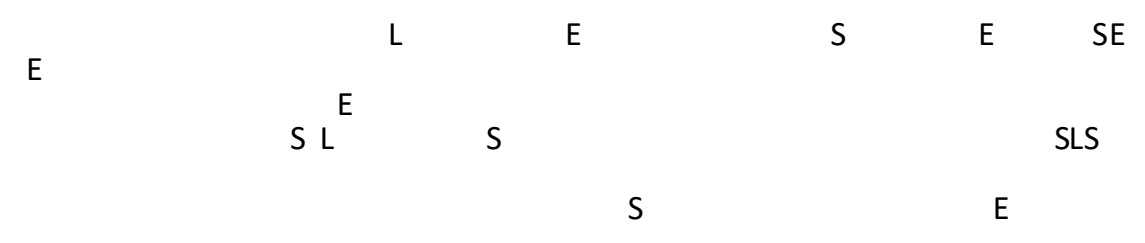

$\mathrm{S}$

E

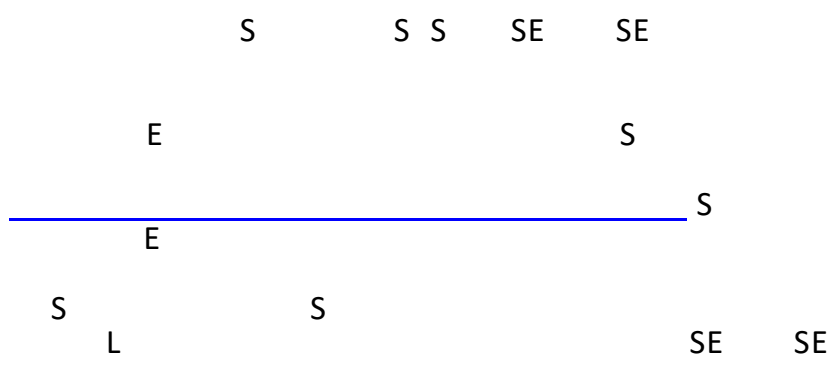

S S SE SE 